\title{
Caveolar vesicles generate DNA damage and perpetuate cellular aging
}

\author{
Keith Wheaton ${ }^{1}$ \\ ${ }^{I}$ Department of Biology, York University, 4700 Keele Street, Toronto, Ontario M3J 1P3, Canada \\ Cell Research (2011) 21:993-994. doi:10.1038/cr.2011.73; published online 26 April 2011
}

The replicative limit of human fibroblasts has long provided a model to assess the molecular mechanisms underlying cellular aging [1]. In culture, fibroblasts which reach the end of their proliferative lifespan acquire profound molecular changes that limit their response to growth factors, and cause permanent exit from the cell cycle [2]. Part of the senescence programme is due to a well-established link between telomere attrition, which occurs with each population doubling and the subsequent upregulation of activity of the p53 tumour suppressor with its transcriptional targets. Critical shortening of telomeres is thought to cause a form of DNA damage, which leads to the activation of caretaker proteins ATM, ATR or DNA-PK that activate $\mathrm{p} 53$, leading to the initiation of senescence through p53 effector genes. In addition, p53 mediates senescence by many other stimuli including oxidative stress, DNA damaging agents and oncogenic activation [3]. About a decade ago, the ectopic expression or endogenous upregulation of caveolin was also shown to lead to p53-mediated senescent arrest [4]. Caveolin is one of the main scaffolding proteins driving the formation of caveolae (50-100 $\mathrm{nm}$ wide cave like invaginations at the plasma

Correspondence: Keith Wheaton

E-mail: kwheaton@yorku.ca membrane) from lipid rafts and allows the organization of many signaling cascades. This compartmentalization concentrates receptors, proteins with lipid anchors, and the lipids from which second messengers are derived. In this capacity, caveolin has been shown to bind and inactivate many key components of mitogenic pathways through the caveolin scaffolding domain (CSD) and thus is often considered as a tumour suppressor [5].

In this issue of Cell Research, Bai et al. [6] explored the connection between caveolar structure and the development of the senescent phenotype. The original investigations into the relationship between caveolae and senescence showed a unexpected upregulation of the proteins caveolin- 1 and 2 during replicative senescence [7, 8]. It was unclear what caused this upregulation, and now Bai et al. demonstrated that it is due to the regulatory cavin protein, PTRF, which is known to drive the biogenesis of caveolae [9]. It is still uncertain what could cause PTRF expression, but it is clearly senescence specific. Furthermore, using electron microscopy (EM), they show convincing upregulation of caveolar structures in senescent cells. In addition to caveolar biogenesis, they demonstrated that PTRF expression also leads to upregulation of Caveolin 1, p53 activation and remarkably DNA damage. The authors concluded from these observations that p53-mediated senes- cence is correlated with the appearance of caveolar structures.

Previous studies were at odds as to whether caveolin expression generated bona-fide functional caveolar structures [7] or represented a differential regulation of lipid rafts [8]. Interestingly, a close examination of the EM of caveolar structures (Figure 6 of Bai's paper [6]), revealed that they are caveolar vesicles and not cave like structures at the membrane. These pinched off vesicles may represent a unique misregulation of caveolae in senescent cells that could explain both the functional differences and the increase in caveolar structures observed previously $[7,8]$. This misregulation may also explain why PTRF leads to DNA damage. Although Bai et al. imply that caveolar structures may directly activate $\mathrm{p} 53$, the most frequently-studied mechanisms to activate $\mathrm{p} 53$ are by the induction of DNA damage. This implies that DNA damage is directly downstream of caveolar vesicle formation, and that the DNA damage causes $\mathrm{p} 53$ activation. Although the most common interpretation in the literature is that telomere attrition is the origin of DNA damage in replicative senescence [3], a considerable amount of damage foci $(\gamma \mathrm{H} 2 \mathrm{AX})$ are not localized to telomeres in senescent cells [10]. The $\gamma \mathrm{H} 2 \mathrm{AX}$-telomere foci are also dependent on whether these cells are cultured in normoxic $\left(2 \% \mathrm{O}_{2}\right)$ conditions [11]. Thus, the possibility 
exists that other forms of stress cause DNA damage in parallel with telomere erosion. One such form of stress may result from the misregulation of caveolae. Caveolar structures are well-known to harbor a great number of signaling cascades that direct cellular proliferation [5] and thus are likely downregulated during senescence [2]. One of these, the epidermal growth factor receptor (EGFR), is well-known to influence the resolution of $\gamma \mathrm{H} 2 \mathrm{AX}$ damage foci and in fact a class of radio-sensitizing agents function through this pathway by antagonizing EGFR [12]. Such drugs enhance the induction of DNA damage and lead to apoptosis of malignant cells through caveolae-mediated EGFR endocytosis. Although primary fibroblasts are genetically stable, they experience transient DNA damage foci as a result of mitogenic stimulation [13]. Therefore, the well-known antagonism of the EGFR by caveolin [7] could perpetuate the normally transient DNA damage foci in fibroblasts [13]. The blocking of EGF signaling in this case would prevent the resolution of damage induced by mitogenic stress during senescence. Another possibility is that DNA damage could be caused by the presence of reactive oxygen species (ROS) produced in cells that overexpress caveolin 1. It has been reported that increased levels of caveolin block thioredoxin reductase 1 activity and that this raises the ROS levels within the fibroblasts tested [14]. Elevated ROS production is well-known to damage DNA, activate p53 and lead to senescence [3]. This pathway may be further augmented by caveolin 1 mediated inactivation of MDM2 and PP2A$\mathrm{C}$, which act as negative regulators of p53 and ATM, respectively [15]. Thus, the negative regulation by caveolin 1 of many key regulatory proteins involved in the DNA damage response could cause DNA damage foci and ensure that signals that lead to a senescent outcome are reinforced.

The study of Bai et al. represents a fundamental shift in our understanding of how the DNA damage occurring in senescence is generated. Remarkably, the increase of caveolar vesicles observed in the senescent state can itself lead to the generation of DNA damage foci in parallel to the well known DNA damage localized to eroded telomeres. The exact mechanism by which this is achieved is still speculative, but likely involves the strong inhibitory activities of the scaffolding protein caveolin 1 . Thus, caveolar vesicles may play an essential role in sequestering and inhibiting key components that normally prevent senescence.

\section{References}

1 Hayflick L. The limited in vitro lifetime of human diploid cell strains. Exp Cell Res 1965; 37:614-636.

2 Wheaton K, Atadja P, Riabowol K. Regulation of transcription factor activity during cellular aging. Biochem Cell Biol 1996; 74:523-534.

3 Ben-Porath I, Weinberg RA. The signals and pathways activating cellular senescence. Int $J$ Biochem Cell Biol 2005; 37:961-976.

4 Galbiati F, Volonte D, Liu J, et al. Caveolin-1 expression negatively regulates cell cycle progression by inducing $\mathrm{G}(0) / \mathrm{G}(1)$ arrest via a $\mathrm{p} 53 / \mathrm{p} 21$ (WAF $1 /$ Cip1)-dependent mechanism. Mol Biol Cell 2001; 12:2229-2244.

5 Zajchowski LD, Robbins SM. Lipid rafts and little caves. Compartmentalized signalling in membrane microdomains. Eur J Biochem 2002; 269:737752.

6 Bai L, Deng X, Li J, et al. Regulation of cellular senescence by the essential caveolar component PTRF/Cavin-1. Cell Res 2011; 21:1088-1101.

7 Park WY, Park JS, Cho KA, et al. Up-regulation of caveolin attenuates epidermal growth factor signaling in senescent cells. J Biol Chem 2000; 275:20847-20852.

8 Wheaton K, Sampsel K, Boisvert FM, et al. Loss of functional caveolae during senescence of human fibroblasts. $J$ Cell Physiol 2001; 187:226-235.

9 Hansen CG, Nichols BJ. Exploring the caves: cavins, caveolins and caveolae. Trends Cell Biol 2010; 20:177-186.

10 Sedelnikova OA, Horikawa I, Zimonjic DB, et al. Senescing human cells and ageing mice accumulate DNA lesions with unrepairable double-strand breaks. Nat Cell Biol 2004; 6:168-170.

11 Herbig U, Jobling WA, Chen BP, Chen DJ, Sedivy JM. Telomere shortening triggers senescence of human cells through a pathway involving ATM, p53, and $\mathrm{p} 21(\mathrm{CIP} 1)$, but not p16(INK4a). Mol Cell 2004; 14:501-513.

12 Meyn RE, Munshi A, Haymach JV, Milas L, Ang KK. Receptor signaling as a regulatory mechanism of DNA repair. Radiother Oncol 2009; 92:316-322.

13 McManus KJ, Hendzel MJ. ATMdependent DNA damage-independent mitotic phosphorylation of $\mathrm{H} 2 \mathrm{AX}$ in normally growing mammalian cells. Mol Biol Cell 2005; 16:5013-5025.

14 Volonte D, Galbiati F. Inhibition of thioredoxin reductase 1 by caveolin 1 promotes stress-induced premature senescence. EMBO Rep 2009; 10:13341340.

15 Volonte D, Galbiati F. Caveolin-1, cellular senescence and pulmonary emphysema. Aging (Albany NY) 2009; 1:831-835. 